\title{
Interpolación de splines cúbicos para estimaciones en elaboración de tablas de mortalidad para Honduras
}

\author{
Christian Samuel Canizales Miranda ${ }^{1}$ \\ Universidad Tecnológica Centroamericana, Tegucigalpa, Honduras.
}

(Enviado: Marzo, 2016. Aceptado para publicación: Noviembre, 2016)

\section{Resumen:}

El instrumento básico para el estudio cuantitativo de la supervivencia es conocido como Tabla de Mortalidad o Tabla de Vida. Las tablas de mortalidad son un modelo teórico de los hechos de mortalidad vividos por una cohorte hipotética de nacidos en una misma fecha, desde el momento inicial del nacimiento hasta la extinción completa de la cohorte por la exclusiva acción de la mortalidad. Las tablas de mortalidad son una variable esencial para la determinación de las reservas para las pensiones como de los recursos requeridos para mantener el principio básico de la sostenibilidad financiera del sistema en el mediano y largo plazo. El método de estimación de tablas presentado en esta investigación, no pretende sustituir los métodos convencionales de elaboración de tablas de mortalidad, es simplemente para estimar las tablas cuando se dispone de poca información. Pero se espera que en el mediano plazo el país tome conciencia de la importancia de las mismas, y trabaje con todas las organizaciones para la recolección de información adecuada y poder elaborar las tablas de la forma convencional que resulta ser la que mejor describe las probabilidades del grupo en observación. Si los gobiernos desean realmente estimular una mejora social para las personas en edad de retiro, y que las personas mayores tengan jubilaciones apropiadas es importante que se elaboren tablas adecuadas para garantizar la estabilidad del sistema.

Palabras Claves: Mortalidad, Interpolación, Supervivencia, Pensiones.

\begin{abstract}
:
The basic tool for the quantitative study of survival is known as mortality table or life table. Mortality tables are a theoretical model of mortality experienced by a hypothetical cohort of those born on the same date, from the initial moment of birth until the complete extinction of the cohort by the exclusive action of mortality. Life tables are essential for determining the reserves for pensions and the resources required to preserve the basic principle of financial stability in the medium and long term. The method presented in this research is not intended to replace conventional ways for mortality tables, it`s just to make estimates when enough information is not available in normal ways. But it is expected on the short term that the country will take awareness on the importance of life tables and work with organizations to collect adequate information and to prepare them in the conventional way. If governments really want to encourage social improvement, for people at retirement age, appropriate life tables should be developed to guarantee the system's stability.
\end{abstract}

Keywords: Mortality, Interpolation, Premium, Survival, Pensions.

${ }^{1}$ Autor para correspondencia. Email: christiancanizales@hotmail.com 


\section{Introducción}

El instrumento básico para el estudio cuantitativo de la supervivencia es conocido como Tabla de Mortalidad o Tabla de Vida. Las tablas de mortalidad son un modelo teórico de los hechos de mortalidad vividos por una cohorte hipotética de nacidos en una misma fecha, desde el momento inicial del nacimiento hasta la extinción completa de la cohorte por la exclusiva acción de la mortalidad. La tabla de mortalidad permite medir las probabilidades de vida y de muerte de una población a una determinada edad.

Las tablas de mortalidad son importantes para la planificación actuarial. La planificación actuarial es el instrumento que permite vigilar la solvencia de los seguros en general, pero más importante, de los sistemas de previsión social. Uno de los objetivos principales dentro de la planificación actuarial es determinar la prima que cumpla con el equilibrio que debe existir entre los recursos disponibles y los gastos.

La planificación actuarial permite a los sistemas de seguridad social determinar las primas necesarias para los futuros beneficios que se van a recibir. En Honduras el tema ha tomado relevancia en los últimos años, principalmente con las nuevas leyes que permiten que los cotizantes puedan tener sus aportaciones en el Régimen de Aportaciones Privadas (RAP) o en cualquier otra AFP del país. Las tablas de mortalidad tienen un papel muy importante porque en base a ellas se debe estimar las primas y reservas necesarias para poder hacer frente a las obligaciones futuras de la AFP o aseguradora.

\section{Descripción de problema}

El problema en Honduras sobre este tema es que no existen tablas de mortalidad diseñadas para la seguridad social con base en la experiencia del país, las tablas utilizadas son de otros países como Estados Unidos para los seguros de vida, Vida Individual Serie CSO 80 y Vida Grupo Tabla CSG 60, y para rentistas se utiliza la Tabla Colombiana 80-89. (Comisión Nacional de Bancos y Seguros, 2015). Para poder desarrollar un sistema de jubilaciones efectivo y poder incluir a todas las personas, se deben tener tablas de mortalidad que reflejen la realidad de la mortalidad del país y puedan utilizarse para rentas vitalicias y seguros de vida, para poder determinar las primas y pagos de jubilación que se deberán realizar a los pensionados.

\subsection{Objetivo General}

El objetivo es poder elaborar tablas con base a las estadísticas de Honduras y no utilizar tablas internaciones. Las tablas deben estar divididas en hombres y mujeres para reflejar las probabilidades de vida para cada grupo ya que son diferentes. El objetivo de la investigación es proporcionar un método que se adapte a la realidad del país, ya que en Honduras se cuenta 
con poca información estadística para realizar las tablas de forma convencional, es necesario encontrar un método alternativo para elaborarlas con la información disponible.

\subsection{Objetivos Específicos}

1. Procesar la información disponible para poder estimar la mortalidad a diferentes edades.

2. Construir la tabla de mortalidad con la información estimada.

3. Determinar un método de ajuste adecuado para la tabla de mortalidad.

\section{Marco Teórico}

La supervivencia humana se relaciona directamente con diferentes factores y algunos pueden ser económicos, sociales, culturales, demográficos y cada uno de ellos con mayor o menor grado de complicación, lo que no se puede negar es la certeza de que el individuo habrá de fallecer, aunque el momento de la ocurrencia de este hecho está definido por la incertidumbre. Asumiremos que la supervivencia de un individuo de la población afecta el tamaño de la misma únicamente por la acción de la mortalidad, quedando excluida las condiciones de movimientos migratorios, por lo que la población en estudio se considera como una población cerrada es decir que no se consideraran las migraciones para el análisis.

Los estudios de mortalidad pueden tener diferentes enfoques, Mazzeo (2014) propone tres tipos de enfoques: "1) El análisis de la mortalidad como componente del crecimiento natural de la población. 2) La construcción de tablas de mortalidad 3) El análisis de los factores que intervienen en el comportamiento de la mortalidad a lo largo del tiempo." (p. 3), la presente investigación se centrará en la construcción de tablas de mortalidad. La tabla de mortalidad es un instrumento que permite medir las probabilidades de vivir y de morir de una población a una edad exacta " $\mathrm{x}$ " o para un grupo de edades entre " $\mathrm{x}$ " $\mathrm{y}$ " $\mathrm{x}+\mathrm{n}$ ". Las tablas de mortalidad describen el proceso de extinción por muerte de una cohorte hipotética de 100,000 nacimientos sometidos a las tasas de mortalidad por edad de la población real para la cual se construye la tabla.

Las variables aleatorias son aquellas cuyo valor numérico se determina al azar, considerando que la certeza del fallecimiento existe, se considera una variable aleatoria la edad del fallecimiento. La edad de ocurrencia del evento está rodeada de incertidumbre, aunque existe la certeza del fallecimiento, por lo que el evento podría considerarse como una distribución binomial en las diferentes edades. Otro aspecto que se asume es la independencia entre las muertes, donde las tasas brutas de mortalidad estarán estimadas por $d^{Z}=\frac{D^{Z}}{N^{30-06-Z}} *$ 1000 donde $\mathrm{d}^{\mathrm{Z}}$ : tasa bruta de mortalidad del año $\mathrm{z}, \mathrm{D}^{\mathrm{Z}}$ : defunciones generales ocurridas en 
el año z, N $\mathrm{N}^{30-06-Z}$ : población estimada al 30 de junio del año z (población media). Asumimos que al estimar las tasas brutas de mortalidad para menores de un año solamente se toman en cuenta los nacidos vivos.

Después de las estimaciones de tasas brutas se calculan las probabilidades de muerte de la población, luego es necesario un proceso graduación para tener una curva suave y poder realizar los cálculos e inferencias necesarios. Según London (1985) citado por Cardozo y Zarruk (2010): "el proceso de graduación es necesario porque la serie de probabilidades de muerte crudas generalmente presenta cambios bruscos, lo cual no corresponde a la hipótesis plausible de que las probabilidades de muerte para dos edades consecutivas deben ser cercanas." (p. 22), los métodos de graduación pueden clasificarse en dos tipos fundamentales, paramétricos y no paramétricos, según se ajuste una función matemática a los datos o simplemente se realice una graduación de los mismos.

Las tablas de mortalidad son la base de la planeación actuarial, por ese motivo es importante tener información actualizada y que refleje las probabilidades reales del país. Como mencionan Cardozo y Zarruk (2010) "Muchos factores han influido para que se presente este incremento en la esperanza de vida y remarcablemente se deben destacar, entre otros, los avances en medicina, así como mayores estándares de vida y mejores sistemas salud pública." (p. 20), es por eso que actualizar las tablas de mortalidad periódicamente es una necesidad, $\mathrm{y}$ tener tablas aplicables a la realidad nacional es necesario para buenas estimaciones.

\section{Metodología}

\subsection{Tipo de Estudio}

Es una investigación aplicada, que tiene un enfoque cualitativo y cuantitativo, es no experimental y explicativo con un enfoque prospectivo.

\subsection{Fuentes de datos, herramientas de recolección utilizadas y procesamiento}

Las fuentes de datos para el análisis son principalmente de informes publicados por los organismos encargados de las diferentes áreas que conforman la investigación, como la SESAL, INE, OIT, OPS, etc. Por lo que la fuente de datos del estudio es documental. El procesamiento de datos es matemático, por medio de fórmulas estadísticas, actuariales y demográficas principalmente. También se procesaron los datos computacionalmente por medio de aplicaciones como Excel y programas estadísticos como MINITAB.

\section{Interpolación de Splines cúbicos para estimaciones en elaboración de tablas de mortalidad para Honduras}




\subsection{Estimación de la mortalidad por género y edad}

Uno de los problemas que se enfrenta en la elaboración de las tablas de mortalidad es la falta de información disponible, en honduras el último censo de población es del 2001. "Los Censos en Honduras datan desde 1791, realizado por la Iglesia Católica. En la actualidad se han realizado XVI Censos de Población siendo el último el del año 2001.” (INE, 2015), y la información de los censos no refleja lo necesario para la elaboración de las tablas, ya que mucha de la información no está agrupada por edades. Es por eso que se tienen que utilizar métodos estadísticos para realizar las inferencias sobre la población objeto de estudio, para esto se utilizaran fuentes de información que procesan estadísticas como tasas brutas de mortalidad a determinadas edades y a partir de eso se calcularan las edades faltantes.

Estas tasas se calculan por lo general para un bienio o trienio, sobre la última información censal. El problema que se presenta es que no se dispone de la información por año de las defunciones por grupos de edad. Sin embargo, tenemos tasas de mortalidad a edades específicas, que pueden servir para pronosticar las edades faltantes por medio de métodos matemáticos. La mortalidad a una edad específica se calcula con la siguiente formula: ${ }_{n} m_{x}{ }^{Z}=\left(\frac{{ }_{n} D_{x}{ }^{Z}}{{ }_{n} N_{x}}\right) * 1000$ dónde: ${ }_{n} \mathrm{~m}_{\mathrm{x}}{ }^{\mathrm{Z}}$ : tasa de mortalidad entre las edades $\mathrm{x} \mathrm{y} \mathrm{x}+\mathrm{n}-1$ del año $\mathrm{z},{ }_{n} \mathrm{D}_{\mathrm{x}} \mathrm{Z}$ : defunciones entre las edades $\mathrm{x}$ y $\mathrm{x}+\mathrm{n}-1$ en el año $\mathrm{z},{ }_{\mathrm{n}} \mathrm{N}_{\mathrm{x}}{ }^{30-06-Z}$ : población con edades entre $x$ y $x+n-1$ estimada al 30 de junio del año $z$.

Sabemos con certeza que al final del periodo de la tabla de mortalidad toda la población estará extinta. La edad final de la tabla se denota por la letra griega $\omega$, por lo que en este caso $\omega=99$. También sabemos que la población hipotética inicial se definió en 100,000 personas que morirán a diferentes edades hasta que la población completa se extinga, por lo tanto, al final de los 99 años el 100\% de la población estará extinta por completo, las probabilidades que la población alcance más de 99 años es mínima por lo que no se consideraran edades mayores a esta edad. Para las tablas de rentistas utilizadas para jubilación las edades finales comúnmente están entre los 100 y los 110 años, pero inician a la edad de 15 años ya que es poco probable que alguien menor realice aportes para jubilación antes de esa edad.

El método elegido para la estimación de los puntos faltantes es el de Splines Cúbicos, se eligió este método porque resulta más conveniente dividir el intervalo en subintervalos más pequeños y usar en cada uno polinomios de grado relativamente bajo, tratando de que la función a trozos definida de este modo tenga un aspecto final adecuado al fenómeno que estamos representando. La idea central es que, en lugar de usar un solo polinomio para interpolar los datos, podemos usar segmentos de polinomios y unirlos adecuadamente para formar la interpolación.

Para definir el Spline de grado $k$ supongamos la siguiente tabla de datos (Tabla 1): 
Tabla 1: Tabla para definir Spline.

\begin{tabular}{|c|cccc|}
\hline $\boldsymbol{x}$ & $x_{0}$ & $x_{1}$ & $\ldots$ & $x_{n}$ \\
\hline $\boldsymbol{y}$ & $y_{0}$ & $y_{1}$ & $\ldots$ & $y_{n}$ \\
\hline
\end{tabular}

Donde suponemos que $\mathrm{x}_{0}<\mathrm{x}_{1}<\ldots<\mathrm{x}_{\mathrm{n}}$, $\mathrm{y}$ dado $k$ un número entero positivo, una función de interpolación Spline de grado $k$, para la tabla de datos, es una función $\mathrm{S}(\mathrm{x})$ tal que: $\mathrm{S}\left(\mathrm{x}_{\mathrm{i}}\right)=\mathrm{y}_{\mathrm{i}}$, para toda $\mathrm{i}=0,1, \ldots, \mathrm{n} . \mathrm{S}(\mathrm{x})$ es un polinomio de grado $\leq k$ en cada subintervalo $\left[\mathrm{x}_{\mathrm{i}-1}, \mathrm{x}_{\mathrm{i}}\right] . \mathrm{S}(\mathrm{x})$ tiene derivada continua hasta de orden $k-1$ en $\left[\mathrm{x}_{0}, \mathrm{x}_{\mathrm{n}}\right]$.

Una Spline Cúbica que interpola la tabla de datos, se define como:

$$
S(x)=\left\{\begin{array}{rll}
S_{0}(x) & \text { si } & x \in\left[x_{0}, x_{1}\right] \\
S_{1}(x) & \text { si } x \in\left[x_{1}, x_{2}\right] \\
S_{2}(x) & \text { si } x \in\left[x_{2}, x_{3}\right] \\
& \text { : } \\
S_{n-1}(x) & \text { si } x \in\left[x_{n-1}, x_{n}\right]
\end{array}\right.
$$

En forma general, dada una función $f$ definida en [a, b] y un conjunto de números, llamados los nodos: $a=\mathrm{x}_{0}<\mathrm{x}_{1}<\ldots<\mathrm{x}_{\mathrm{n}}=\mathrm{b}$, un interpolante cúbico de trazador $\mathrm{S}$ para $f$ es una función que satisface las siguientes condiciones:

a) $\mathrm{S}$ es un polinomio cúbico, denotado $\mathrm{S}_{\mathrm{j}}$, en el subintervalo $\left[\mathrm{x}_{\mathrm{j}}, \mathrm{x}_{\mathrm{j}-1}\right]$ para cada $j=0,1, \ldots, n-1$;

b) $\mathrm{S}\left(\mathrm{x}_{\mathrm{j}}\right)=f\left(\mathrm{x}_{\mathrm{j}}\right)$ para cada $j=0,1, \ldots, n$;

c) $\mathrm{S}_{\mathrm{j}+1}\left(\mathrm{x}_{\mathrm{j}+1}\right)=\mathrm{S}_{\mathrm{j}}\left(\mathrm{x}_{\mathrm{j}+1}\right)$ para cada $j=0,1, \ldots, n-2$;

d) $\mathrm{S}_{\mathrm{j}+1}^{\prime}\left(\mathrm{x}_{\mathrm{j}+1}\right)=\mathrm{S}_{\mathrm{j}}^{\prime}\left(\mathrm{x}_{\mathrm{j}+1}\right)$ para cada $j=0,1, \ldots, n-2$

e) $\mathrm{S}_{\mathrm{j}+1}\left(\mathrm{x}_{\mathrm{j}+1}\right)=\mathrm{S}_{\mathrm{j}}\left(\mathrm{x}_{\mathrm{j}+1}\right)$ para cada $j=0,1, \ldots, n-2$;

f) Se satisface una, del siguiente conjunto de condiciones de frontera:

$$
\begin{aligned}
& \mathrm{S}^{\prime \prime}\left(\mathrm{x}_{0}\right)=\mathrm{S}^{\prime \prime}\left(\mathrm{x}_{\mathrm{n}}\right)=0(\text { frontera libre) } \\
& \mathrm{S}^{\prime}\left(\mathrm{x}_{0}\right)=f^{\prime}\left(\mathrm{x}_{0}\right) \text { y } \mathrm{S}^{\prime}\left(\mathrm{x}_{\mathrm{n}}\right)=f^{\prime}\left(\mathrm{x}_{\mathrm{n}}\right) \text { (frontera sujeta) }
\end{aligned}
$$


La ecuación b) indica que el Spline cúbico se ajusta a cada uno de los puntos, que es continua c), y que es continua en pendiente y curvatura d) y e), a lo largo de toda la región generada por los puntos. Para construir el interpolante cúbico de trazador para una función $f$ dada, se pueden aplicar las condiciones de la definición a los polinomios cúbicos.

$\mathrm{S}_{j}(\mathrm{x})=a_{j}+b_{j}\left(x-x_{j}\right)+c_{j}\left(x-x_{j}\right)^{2}+d_{j}\left(x-x_{j}\right)^{3}$ para cada $j=0,1, \ldots, n-1$

Para $x=x_{j}: \mathrm{S}_{j}\left(x_{j}\right)=a_{j}=f\left(x_{j}\right)$ y se aplica la condición c):

$$
\begin{aligned}
& a_{j+1}= \mathrm{S}_{j+1}\left(x_{j+1}\right)=\mathrm{S}_{j}\left(x_{j+1}\right)=a_{j}+b_{j}\left(x_{j+1}-x_{j}\right)+c_{j}\left(x_{j+1}-x_{j}\right)^{2}+d_{j}\left(x_{j+1}-x_{j}\right)^{3} \\
& \quad \text { para cada } j=0,1, \ldots, n-2
\end{aligned}
$$

Con la notación $h_{j}=x_{j+1}-x_{j}$ para cada $j=0,1, \ldots, n-1$ y se define $a_{n}=f\left(x_{n}\right)$ entonces:

$a_{j+1}=a_{j}+b_{j} h_{j}+c_{j} h_{j}{ }^{2}+d_{j} h_{j}^{3}$ para cada $j=0,1, \ldots, n-1$.

Definiendo $b_{n}=S^{\prime}\left(x_{n}\right)$ se tiene:

$$
\begin{aligned}
S^{\prime} & (x)=b_{j}+2 c_{j}\left(x-x_{j}\right)+3 d_{j}\left(x-x_{j}\right)^{2} \operatorname{para} x=x_{j}: S^{\prime}{ }_{j}\left(x_{j}\right)=b_{j} \\
& \text { para cada } j=0,1, \ldots, n-1 .
\end{aligned}
$$

Aplicando la condición d) se tiene:

$b_{j+1}=S_{j+1}^{\prime}\left(x_{j+1}\right)=S^{\prime}{ }_{j}\left(x_{j+1}\right)=b_{j}+2 c_{j} h_{j}+3 d_{j} h_{j}{ }^{2}$ para cada $j=0,1, \ldots, n-1$.

Aplicando la condición e), si se define, $c_{n}=\frac{s^{\prime \prime}\left(x_{n}\right)}{2}$ entonces:

$c_{j+1}=S^{\prime \prime}{ }_{j+1}\left(x_{j+1}\right)=S^{\prime \prime}{ }_{j}\left(x_{j+1}\right)=c_{j}+3 d_{j} h_{j}$ para cada $j=0,1, \ldots, n-1$.

Despejando $d_{j}$ en la ecuación anterior: $d_{j}=\frac{\left(c_{j+1}-c_{j}\right)}{3 h_{j}}$ sustituyendo $d_{j}$ en $b_{j+1}$ y operando tenemos:

$b_{j+1}=b_{j}+\left(c_{j}+c_{j+1}\right) h_{j}$ reduciendo el índice en $b_{j+1}$ se tiene:

$$
b_{j}=b_{j-1}+\left(c_{j-1}+c_{j}\right) h_{j-1}
$$


Sustituyendo $d_{j}$ en $a_{j+1}$ y operando se tiene: $a_{j+1 .}=a_{j}+b_{j} h_{j}+\frac{h_{j}^{2}}{3}\left(2 c_{j}+c_{j+1}\right)$ despejando para $b_{j}$ y operando se tiene: $b_{j}=\frac{\left(a_{j+1}-a_{j}\right)}{h_{j}}-\frac{h_{j}}{3}\left(2 c_{j}+c_{j+1}\right)$ reduciendo el índice en $b_{j}$ se tiene:

$$
\begin{aligned}
& b_{j-1 .}=\frac{\left(a_{j .}-a_{j-1}\right)}{h_{j-1}}-\frac{h_{j-1}}{3}\left(2 c_{j-1}+c_{j}\right) \text { sustituyendo } b_{j .} \text { y } b_{j-1 .} \text { en } b_{j} \text { se tiene: } \\
& \frac{\left(a_{j+1}-a_{j}\right)}{h_{j}}-\frac{h_{j}}{3}\left(2 c_{j}+c_{j+1}\right)=\frac{\left(a_{j}-a_{j-1}\right)}{h_{j-1}}-\frac{h_{j-1}}{3}\left(2 c_{j-1}+c_{j}\right)+\left(c_{j}+c_{j-1}\right) h_{j-1}
\end{aligned}
$$

Operando algebraicamente las ecuaciones anteriores se tiene:

$$
h_{j-1} c_{j-1}+2\left(h_{j-1}+h_{j}\right) c_{j}+h_{j}+c_{j+1}=\frac{3}{h_{j}}\left(a_{j+1}-a_{j}\right)-\frac{3}{h_{j-1}}\left(a_{j}-a_{j-1}\right)
$$

Para cada $j=1,2, \ldots, n-1$. Las incógnitas del sistema son $\left\{c_{j}\right\}_{j=0}^{n}$ porque los valores $\left\{h_{j}\right\}_{j=0}^{n-1}$ y $\left\{a_{j}\right\}_{j=0}^{n-1}$ están definidos por el espacio de los nodos $\left\{x_{j}\right\}_{j=0}^{n}$ y los valores de $f$ en los nodos. Una vez que se conoce los valores de $c_{j}$ encontrar los valores para $b_{j}$ y $d_{j}$ y construir los polinomios cúbicos resulta más fácil al sustituir $c_{j}$ en las ecuaciones anteriores, si se cumplen las condiciones de frontera libre $\mathrm{S}^{\prime \prime}\left(\mathrm{x}_{0}\right)=\mathrm{S}^{\prime \prime}\left(\mathrm{x}_{\mathrm{n}}\right)=0$ implican que $0=\mathrm{S}^{\prime \prime}\left(\mathrm{x}_{0}\right)=2 c_{0}+6 d_{0}\left(x_{0 \text { - }}\right.$ $\left.x_{0}\right) \Rightarrow c_{0}=0$

$c_{n}=\frac{s^{\prime \prime}\left(x_{n}\right)}{2}=0 \Rightarrow c_{n}=0$, las dos ecuaciones $c_{0}=0$ y $c_{n}=0$ junto con la igualdad de ecuaciones anteriores, producen un sistema lineal descrito por la ecuación vectorial $A x=b$ donde $A$ es una matriz de $(n+1)$ por $(n+1)$.

$$
\begin{gathered}
A=\left(\begin{array}{ccccc}
1 & 0 & 0 & \cdots & 0 \\
h_{0} & 2\left(h_{0}+h_{1}\right) & h_{1} & \ddots & \vdots \\
0 & h_{3} & 2\left(h_{1}+h_{2}\right) & h_{2} & 0 \\
\vdots & \ddots & h_{n-2} & 2\left(h_{n-2}+h_{1}\right) & h_{n-1} \\
0 & \cdots & 0 & 0 & 1
\end{array}\right) x=\left[\begin{array}{c}
c_{0} \\
c_{1} \\
c_{2} \\
\vdots \\
c_{n}
\end{array}\right] \\
b=\left[\begin{array}{c}
0 \\
\frac{3}{h_{0}}\left(a_{2}-a_{1}\right)-\frac{3}{h_{0}}\left(a_{1}-a_{0}\right) \\
\frac{3}{h_{n-1}}\left(a_{n}-a_{n-1}\right)-\frac{3}{h_{n-2}}\left(a_{n+1}-a_{n-2}\right) \\
0
\end{array}\right]
\end{gathered}
$$

Una vez definido el método a utilizar se elabora la tabla con la información disponible para luego aplicar la interpolación. Utilizando información del 2013 de tasas brutas de mortalidad, se procede a construir una tabla con las muertes a las diferentes edades, para este ejemplo se 
utilizará solamente la información de hombres, pero el procedimiento para mujeres es el mismo. La información de las tablas proviene de los datos y estadísticas de la Encuesta Nacional de Salud y Demografía (ENDESA), según indicadores de mortalidad (Secretaría de Salud [Honduras], Instituto Nacional de Estadística e ICF International, 2013, p. 171) y de la Organización Mundial de la Salud. (World Health Organization, 2015).

Tabla 2: Fallecimientos acumulados esperados por edad.

\begin{tabular}{|c|c|c|c|c|c|c|c|}
\hline Edad & $\mathbf{0}$ & $\mathbf{5}$ & $\mathbf{1 5}$ & $\mathbf{6 0}$ & $\mathbf{7 0}$ & $\mathbf{8 0}$ & $\mathbf{9 9}$ \\
\hline $\begin{array}{c}\text { Total de } \\
\text { Fallecidos }\end{array}$ & 2,300 & 3,000 & 5,358 & 27,138 & 44,915 & 57,471 & 100,000 \\
\hline
\end{tabular}

La información de la Tabla 2 se obtiene por medio de tasas de mortalidad, es decir la tasa estimada de mortalidad para los niños varones menores de 1 año en el 2013 es de 23 niños por cada mil nacidos vivos, para obtener esta tasa en porcentaje dividimos entre mil y obtenemos $2.3 \%$ al aplicarlo a la población hipotética de 100,000 tenemos como resultado 2,300 fallecimientos esperados para la población menor a un año. Según la organización mundial de la salud la probabilidad de morir entre los 15 y los 60 para los hombres en el año 2013 es de 176 muertes por cada mil habitantes, es decir una probabilidad de 17.6\% (World Health Organization, 2015). El mismo procedimiento se utiliza para las siguientes tasas de mortalidad que se pudieron encontrar, para la edad de 70 años se utiliza la esperanza de vida al nacer de los varones y se espera que aproximadamente el $45 \%$ de la población no supere esta edad y suponemos que a los 99 años toda la población habrá fallecido por lo que a esta edad el total de fallecimientos es 100,000.

A partir de esto se utiliza el método de los Splines Cúbicos para encontrar las ecuaciones que nos ayudaran a encontrar los datos faltantes. Las funciones cúbicas a utilizar serán las siguientes:

$$
S(x)=\left\{\begin{array}{ccc}
a_{1} x^{3}+b_{1} x^{2}+c_{1} x+d_{1} & \text { si } x \in[0,5] \\
a_{2} x^{3}+b_{2} x^{2}+c_{2} x+d_{2} & \text { si } x \in[5,15] \\
a_{3} x^{3}+b_{3} x^{2}+c_{3} x+d_{3} & \text { si } x \in[15,60] \\
a_{4} x^{3}+b_{4} x^{2}+c_{4} x+d_{4} & \text { si } x \in[60,70] \\
a_{5} x^{3}+b_{5} x^{2}+c_{5} x+d_{5} & \text { si } x \in[70,80] \\
a_{6} x^{3}+b_{6} x^{2}+c_{6} x+d_{6} & \text { si } x \in[80,99]
\end{array}\right.
$$

Operando con el sistema de ecuaciones y resolviendo se puede estimar los fallecimientos a las diferentes edades como se muestra en la tabla siguiente: 
Tabla 3: Estimaciones del total de fallecimientos por edades de 0-99 años.

\begin{tabular}{cccccccc}
\hline Edad & Fallecimientos & Edad & Fallecimientos & Edad & Fallecimientos & Edad & Fallecimientos \\
\hline 0 & 2,300 & 25 & 6,351 & 50 & 14,519 & 75 & 51,245 \\
1 & 2,417 & 26 & 6,403 & 51 & 15,435 & 76 & 52,396 \\
2 & 2,540 & 27 & 6,457 & 52 & 16,421 & 77 & 53,568 \\
3 & 2,675 & 28 & 6,516 & 53 & 17,479 & 78 & 54,787 \\
4 & 2,826 & 29 & 6,583 & 54 & 18,613 & 79 & 56,080 \\
5 & 3,000 & 30 & 6,660 & 55 & 19,824 & 80 & 57,471 \\
6 & 3,200 & 31 & 6,750 & 56 & 21,115 & 81 & 58,982 \\
7 & 3,424 & 32 & 6,856 & 57 & 22,489 & 82 & 60,610 \\
8 & 3,664 & 33 & 6,981 & 58 & 23,949 & 83 & 62,350 \\
9 & 3,916 & 34 & 7,126 & 59 & 25,498 & 84 & 64,195 \\
10 & 4,174 & 35 & 7,295 & 60 & 27,138 & 85 & 66,138 \\
11 & 4,432 & 36 & 7,491 & 61 & 28,867 & 86 & 68,173 \\
12 & 4,685 & 37 & 7,715 & 62 & 30,668 & 87 & 70,294 \\
13 & 4,928 & 38 & 7,972 & 63 & 32,517 & 88 & 72,493 \\
14 & 5,154 & 39 & 8,263 & 64 & 34,392 & 89 & 74,764 \\
15 & 5,358 & 40 & 8,592 & 65 & 36,271 & 90 & 77,101 \\
16 & 5,536 & 41 & 8,960 & 66 & 38,129 & 91 & 79,496 \\
17 & 5,690 & 42 & 9,371 & 67 & 39,946 & 92 & 81,945 \\
18 & 5,821 & 43 & 9,828 & 68 & 41,697 & 93 & 84,439 \\
19 & 5,933 & 44 & 10,332 & 69 & 43,361 & 94 & 86,972 \\
20 & 6,029 & 45 & 10,887 & 70 & 44,915 & 95 & 89,538 \\
21 & 6,110 & 46 & 11,496 & 71 & 46,344 & 96 & 92,131 \\
22 & 6,180 & 47 & 12,161 & 72 & 47,666 & 97 & 94,743 \\
23 & 6,242 & 48 & 12,885 & 73 & 48,906 & 98 & 97,368 \\
24 & 6,298 & 49 & 13,670 & 74 & 50,090 & 99 & 100,000 \\
\hline
\end{tabular}

Recordando que a la edad de 99 años el total de la población habrá fallecido, y que los fallecimientos por edad representan el total acumulado a esa edad. Podemos comprobar que los Splines cumplen con los parámetros al observar las estimaciones para las edades proporcionadas en la tabla inicial, para cero un total de 2,300 fallecimientos, para cinco 3,000 , para quince 5,358 y así sucesivamente. Al tener los mismos valores utilizando los polinomios cúbicos de Splines se demuestra que los parámetros se cumplieron, la gráfica para la tabla anterior es la siguiente: 


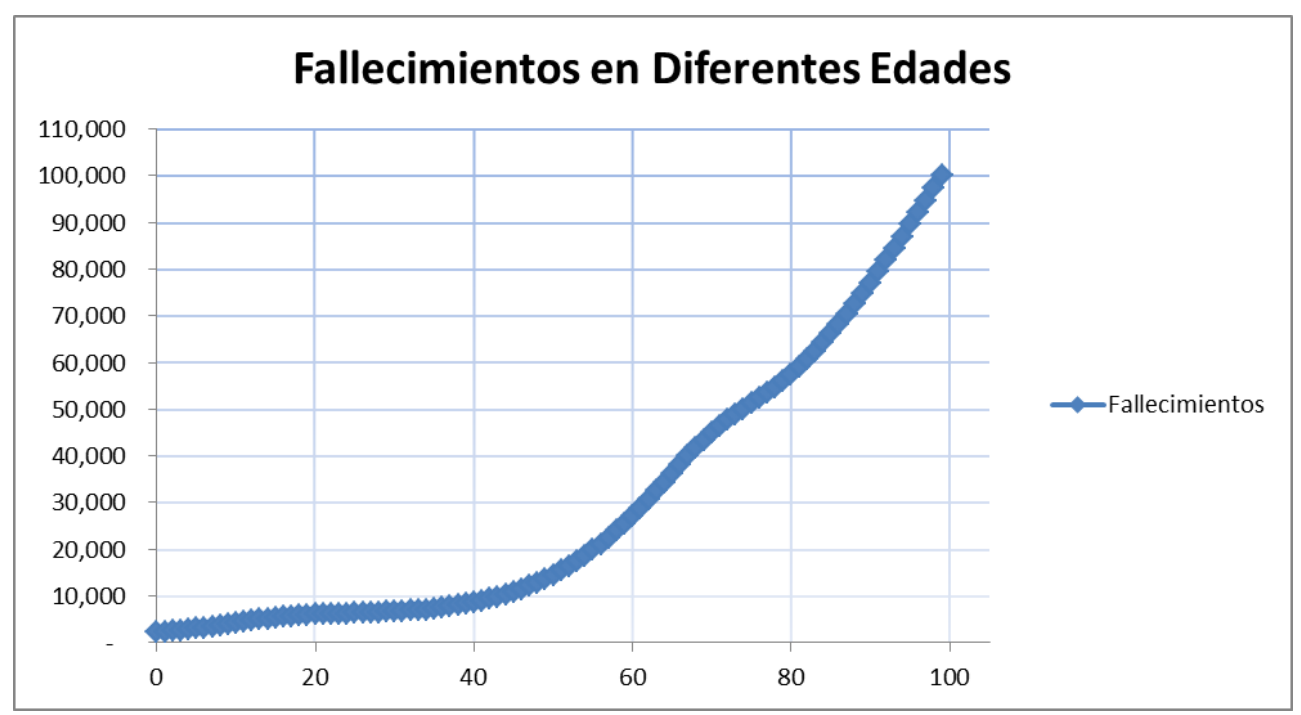

Figura 1: Estimación de fallecimientos por edades de 0-99 años.

A partir de esta información podemos trabajar para construir la tabla de mortalidad.

\subsection{Construcción de la tabla de mortalidad}

Una vez que tenemos los datos de fallecidos a las diferentes edades se puede proceder fácilmente con la estimación de ${ }_{n} q_{x}$ que es la base de las tablas de mortalidad, donde: La primera columna corresponde a la edad, en la que se indica la edad exacta " $x$ " o la edad inicial del grupo de edad. $1_{x}$ : número de personas que alcanza con vida la edad exacta " $x$ ", de una generación inicial de 100.000 nacimientos $\left(l_{0}\right)$. ${ }_{n} d_{x}$ : número de defunciones ocurridas entre las edades exactas " $\mathrm{x}$ " $\mathrm{y}$ " $\mathrm{x}+\mathrm{n}$ ". $\mathrm{nq}_{\mathrm{x}}$ : probabilidad que tiene una persona de edad exacta " $x$ " de fallecer antes de alcanzar la edad exacta " $x+n$ ".

Para calcular ${ }_{n} d_{x}$ proviene de la columna fallecimientos donde: $(x+1)-x$ para obtener el total de fallecimientos por edad. Primero se estima que la probabilidad de morir a determinada edad según los indicadores que se disponen, es decir que se utiliza la probabilidad de ocurrencia del evento a determinada edad, donde la probabilidad total estaría representada por: $\mathrm{P}(\mathrm{A}+\mathrm{B})=\mathrm{P}(\mathrm{A})+\mathrm{P}(\mathrm{B})$, y la probabilidad de presentación conjunta es nula, porque o muere a una edad o muere en otra, pero no puede presentarse al mismo tiempo.

Sabiendo que la probabilidad total de ocurrencia del evento es igual a 1, porque la persona fallecerá en alguna edad entre 0 y $\omega$, y que la probabilidad acumulada de fallecer entre 0-70 años es aproximadamente $45 \%$, y las probabilidades de $0-1$ es de $2.3 \%$, entre $1-5$ de $0.7 \%$ (probabilidad acumulada de cero a cinco es 3\%), probabilidad de morir entre 15-60 es 17.6\%, y estimando que la probabilidad de morir entre 60-70 años es aproximadamente 3.5 veces 
mayor que entre 5-15, se puede plantear la siguiente ecuación para determinar las probabilidades faltantes de 5-15 años y de $60-70$ años: $45 \%=3 \%+x+17.6 \%+3.5 x$ al despejar para $x$ tenemos que $x$ es aproximadamente igual a $5 \%$, por lo que para las edades de 5-15 sería la misma probabilidad y para 60-70 seria aproximadamente igual a 19\%. Según la Organización Mundial de la Salud, la esperanza de vida después de los 60 años para los hombres en Honduras es de 21 años, por lo que se espera que al menos el 57\% de la población fallezca a la edad de 80 años, por lo tanto $57 \%=x+45 \%$ despejando para $x$, es la probabilidad de fallecer entre 70-80 entonces $x=13$. Siendo la probabilidad de fallecer entre 80-99 años igual a $100 \%-57 \%=43 \%$, estas probabilidades se utilizan para elaborar la Tabla 2 y a partir de esto, después de utilizar los splines cúbicos, se calcula $\mathrm{d}_{\mathrm{x}}$ como el total de fallecimientos en $x+1$ menos el total de fallecimientos en $x$ como se muestra en las siguientes tablas:

Tabla 4: Tablas de mortalidad estimada a diferentes edades

\begin{tabular}{cccccccccc}
\hline Edad & Fallecimientos & ${ }_{n} \mathrm{~d}_{\mathrm{x}}$ & $\mathrm{l}_{\mathrm{x}}$ & ${ }_{\mathrm{n}} \mathrm{q}_{\mathrm{x}}$ & $\mathrm{Edad}$ & Fallecimientos & ${ }_{\mathrm{n}} \mathrm{d}_{\mathrm{x}}$ & $\mathrm{l}_{\mathrm{x}}$ & ${ }_{\mathrm{n}} \mathrm{q}_{\mathrm{x}}$ \\
\hline 0 & 2,300 & 2,300 & 100,000 & 0.023000 & 25 & 6,351 & 53 & 93,702 & 0.000561 \\
1 & 2,417 & 117 & 97,700 & 0.001201 & 26 & 6,403 & 52 & 93,649 & 0.000557 \\
2 & 2,540 & 123 & 97,583 & 0.001261 & 27 & 6,457 & 54 & 93,597 & 0.000581 \\
3 & 2,675 & 134 & 97,460 & 0.001378 & 28 & 6,516 & 59 & 93,543 & 0.000634 \\
4 & 2,826 & 151 & 97,325 & 0.001555 & 29 & 6,583 & 67 & 93,484 & 0.000715 \\
5 & 3,000 & 174 & 97,174 & 0.001790 & 30 & 6,660 & 77 & 93,417 & 0.000826 \\
6 & 3,200 & 200 & 97,000 & 0.002066 & 31 & 6,750 & 90 & 93,340 & 0.000966 \\
7 & 3,424 & 223 & 96,800 & 0.002305 & 32 & 6,856 & 106 & 93,250 & 0.001135 \\
8 & 3,664 & 240 & 96,576 & 0.002488 & 33 & 6,981 & 124 & 93,144 & 0.001334 \\
9 & 3,916 & 252 & 96,336 & 0.002615 & 34 & 7,126 & 145 & 93,019 & 0.001563 \\
10 & 4,174 & 258 & 96,084 & 0.002684 & 35 & 7,295 & 169 & 92,874 & 0.001821 \\
11 & 4,432 & 258 & 95,826 & 0.002696 & 36 & 7,491 & 196 & 92,705 & 0.002110 \\
12 & 4,685 & 253 & 95,568 & 0.002649 & 37 & 7,715 & 225 & 92,509 & 0.002430 \\
13 & 4,928 & 242 & 95,315 & 0.002544 & 38 & 7,972 & 257 & 92,285 & 0.002781 \\
14 & 5,154 & 226 & 95,072 & 0.002379 & 39 & 8,263 & 291 & 92,028 & 0.003164 \\
15 & 5,358 & 204 & 94,846 & 0.002154 & 40 & 8,592 & 328 & 91,737 & 0.003581 \\
16 & 5,536 & 178 & 94,642 & 0.001883 & 41 & 8,960 & 368 & 91,408 & 0.004031 \\
17 & 5,690 & 154 & 94,464 & 0.001625 & 42 & 9,371 & 411 & 91,040 & 0.004515 \\
18 & 5,821 & 131 & 94,310 & 0.001394 & 43 & 9,828 & 456 & 90,629 & 0.005036 \\
19 & 5,933 & 112 & 94,179 & 0.001190 & 44 & 10,332 & 504 & 90,172 & 0.005595 \\
20 & 6,029 & 95 & 94,067 & 0.001015 & 45 & 10,887 & 555 & 89,668 & 0.006192 \\
21 & 6,110 & 81 & 93,971 & 0.000867 & 46 & 11,496 & 609 & 89,113 & 0.006830 \\
22 & 6,180 & 70 & 93,890 & 0.000748 & 47 & 12,161 & 665 & 88,504 & 0.007511 \\
23 & 6,242 & 62 & 93,820 & 0.000657 & 48 & 12,885 & 724 & 87,839 & 0.008238 \\
24 & 6,298 & 56 & 93,758 & 0.000595 & 49 & 13,670 & 785 & 87,115 & 0.009013 \\
\hline
\end{tabular}

La gráfica comparativa de la estimación con la tabla que actualmente se utiliza para seguros de individual para hombres Serie CSO 80 y para Vida Grupo Tabla CSG 60 es la siguiente: 

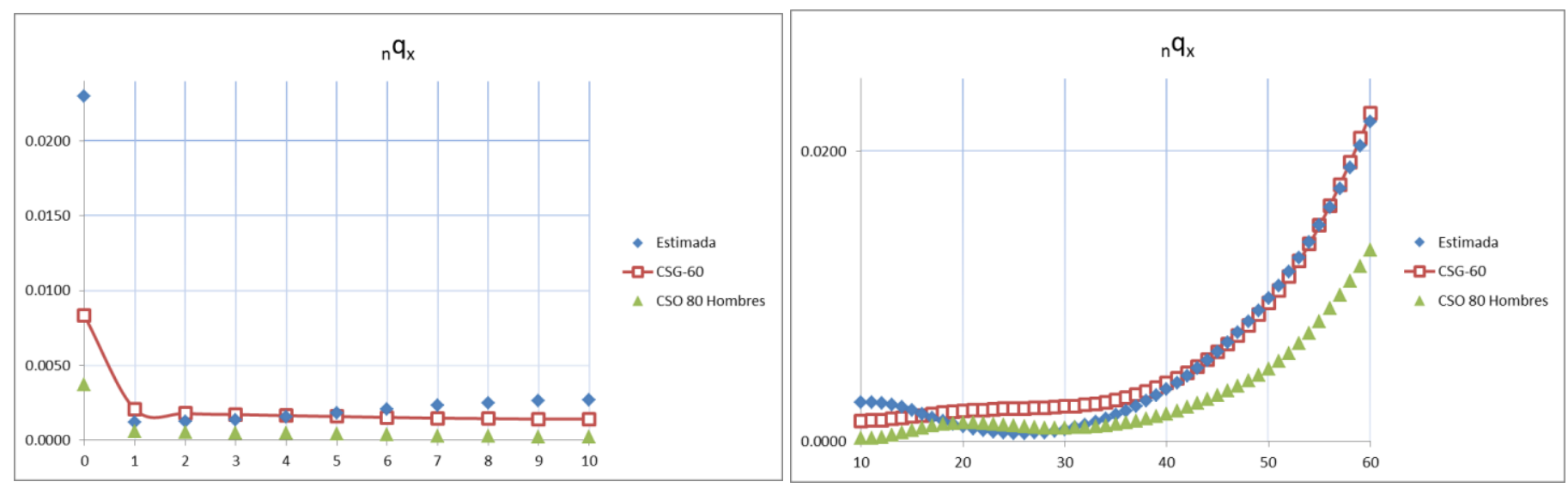

Figura 2. Gráfica comparativa 1

Tabla 5: Continuación de la tabla de mortalidad

\begin{tabular}{|c|c|c|c|c|c|c|c|c|c|}
\hline Edad & Fallecimientos & ${ }_{n} d_{x}$ & $1_{x}$ & ${ }_{n} q_{x}$ & Edad & Fallecimientos & ${ }_{n} d_{x}$ & $1_{x}$ & ${ }_{n} q_{x}$ \\
\hline 50 & 14,519 & 849 & 86,330 & 0.009839 & 75 & 51,245 & 1,155 & 49,910 & 0.023135 \\
\hline 51 & 15,435 & 916 & 85,481 & 0.010719 & 76 & 52,396 & 1,151 & 48,755 & 0.023598 \\
\hline 52 & 16,421 & 986 & 84,565 & 0.011659 & 77 & 53,568 & 1,172 & 47,604 & 0.024621 \\
\hline 53 & 17,479 & 1,058 & 83,579 & 0.012661 & 78 & 54,787 & 1,219 & 46,432 & 0.026262 \\
\hline 54 & 18,613 & 1,133 & 82,521 & 0.013732 & 79 & 56,080 & 1,292 & 45,213 & 0.028587 \\
\hline 55 & 19,824 & 1,211 & 81,387 & 0.014878 & 80 & 57,471 & 1,391 & 43,920 & 0.031679 \\
\hline 56 & 21,115 & 1,291 & 80,176 & 0.016106 & 81 & 58,982 & 1,511 & 42,529 & 0.035518 \\
\hline 57 & 22,489 & 1,374 & 78,885 & 0.017423 & 82 & 60,610 & 1,629 & 41,018 & 0.039703 \\
\hline 58 & 23,949 & 1,460 & 77,511 & 0.018838 & 83 & 62,350 & 1,740 & 39,390 & 0.044175 \\
\hline 59 & 25,498 & 1,549 & 76,051 & 0.020364 & 84 & 64,195 & 1,845 & 37,650 & 0.049003 \\
\hline 60 & 27,138 & 1,640 & 74,502 & 0.022011 & 85 & 66,138 & 1,943 & 35,805 & 0.054275 \\
\hline 61 & 28,867 & 1,729 & 72,862 & 0.023737 & 86 & 68,173 & 2,035 & 33,862 & 0.060100 \\
\hline 62 & 30,668 & 1,801 & 71,133 & 0.025315 & 87 & 70,294 & 2,120 & 31,827 & 0.066622 \\
\hline 63 & 32,517 & 1,849 & 69,332 & 0.026672 & 88 & 72,493 & 2,199 & 29,706 & 0.074026 \\
\hline 64 & 34,392 & 1,875 & 67,483 & 0.027785 & 89 & 74,764 & 2,271 & 27,507 & 0.082566 \\
\hline 65 & 36,271 & 1,878 & 65,608 & 0.028628 & 90 & 77,101 & 2,337 & 25,236 & 0.092595 \\
\hline 66 & 38,129 & 1,859 & 63,729 & 0.029165 & 91 & 79,496 & 2,396 & 22,899 & 0.104621 \\
\hline 67 & 39,946 & 1,816 & 61,871 & 0.029359 & 92 & 81,945 & 2,448 & 20,504 & 0.119403 \\
\hline 68 & 41,697 & 1,752 & 60,054 & 0.029166 & 93 & 84,439 & 2,494 & 18,055 & 0.138136 \\
\hline 69 & 43,361 & 1,664 & 58,303 & 0.028541 & 94 & 86,972 & 2,533 & 15,561 & 0.162804 \\
\hline 70 & 44,915 & 1,554 & 56,639 & 0.027433 & 95 & 89,538 & 2,566 & 13,028 & 0.196980 \\
\hline 71 & 46,344 & 1,429 & 55,085 & 0.025940 & 96 & 92,131 & 2,592 & 10,462 & 0.247806 \\
\hline 72 & 47,666 & 1,322 & 53,656 & 0.024633 & 97 & 94,743 & 2,612 & 7,869 & 0.331945 \\
\hline 73 & 48,906 & 1,240 & 52,334 & 0.023699 & 98 & 97,368 & 2,625 & 5,257 & 0.499376 \\
\hline 74 & 50,090 & 1,185 & 51,094 & 0.023185 & 99 & 100,000 & 2,632 & 2,632 & 1.000000 \\
\hline
\end{tabular}



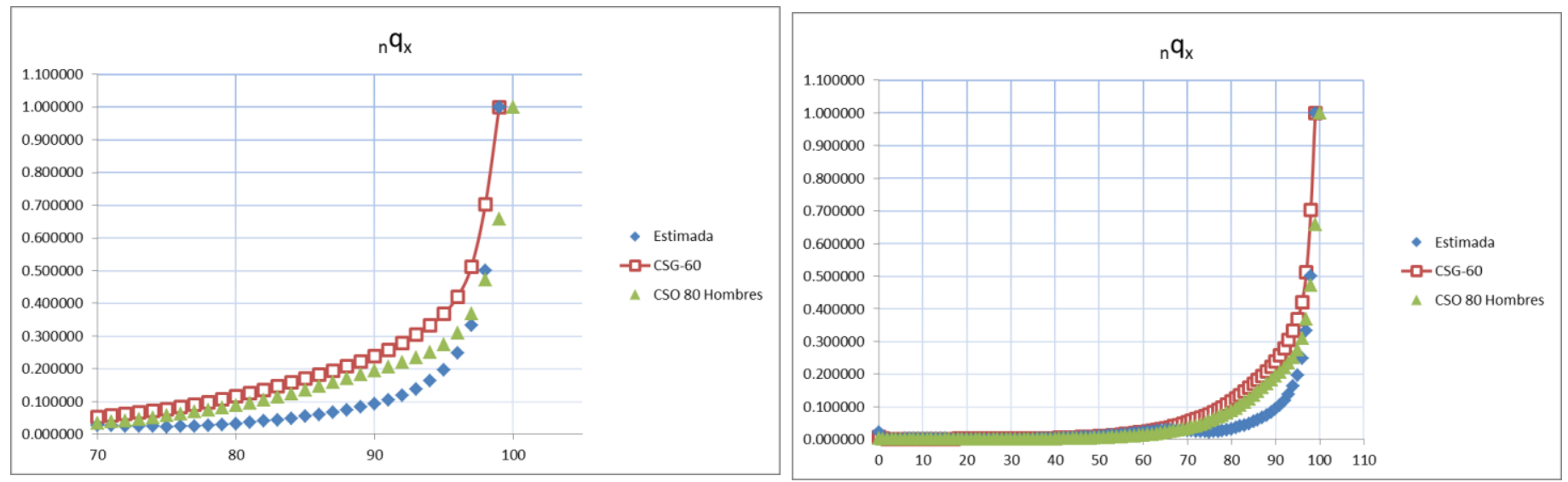

Figura 3: Gráfica Comparativa 2

En las tablas anteriores (Tabla 4 y 5) después de obtener el valor de ${ }_{n} d_{\mathrm{x}}$ se puede calcular $1_{\mathrm{x}}$, iniciando con $1_{0}=100,000$ que es la población hipotética inicial y luego con la formula $1_{\mathrm{x}}=1_{\mathrm{x}}$ ${ }_{1}-{ }_{n} d_{x-1}$, finalmente ${ }_{n} q_{x}$ se calcula con la formula ${ }_{n} q_{x}={ }_{n} d_{x} / 1_{x}$ que sería la probabilidad de fallecer a determinada edad. Como se puede observar en las gráficas anteriores, las variaciones tienen diferencias principalmente en las edades tempranas y finales de la tabla, esto se explica porque en Honduras la mortalidad infantil es mayor que en Estados Unidos, que es de donde provienen las tablas. Esto también explica las diferencias a las edades finales de la tabla, ya que las tablas de Estados Unidos tienen una mayor mortalidad porque es mayor la población que logra alcanzar esta edad que en Honduras. Existen varias versiones de las tablas CSO 80, incluyendo tablas con fumadores, y con diversa información, pero la actualización de las tablas es importante para reflejar la mortalidad real a que está expuesta la población. Las tablas CSO 80 fueron elaboradas en 1980 y desde ese entonces los avances de la medicina hacen necesarias las actualizaciones, también, en Honduras se debe considerar los factores como la violencia, influyen en las probabilidades de morir a edades menores que en otros países.

\subsection{Método de ajuste para la tabla de mortalidad}

El metodo de ajuste elegido en este caso es un metodo no parametrico conocido como Método de Whittaker-Henderson. Como lo plantea Lowrie (1993) en la formulación básica de W-H, los conceptos de suavidad y ajuste se presentan en la ecuación lineal:

$$
M=F+h S=\sum_{x=1}^{n} w_{x}\left(v_{x}-u_{x}\right)^{2}+h \sum_{x=1}^{n-z}\left(\Delta^{2} v_{x}\right)^{2}
$$


Los valores graduados $v_{x}$, para $x=12,3, \ldots, \mathrm{n}$, se eligen de tal manera que minimicen el valor de $M$. El primer término mide la calidad del ajuste, asociándole el peso relativo del tamaño de la muestra a las desviaciones de la graduación. Cuando el resultado de la suma de las desviaciones de la graduación al cuadrado es cercano a cero (0), estaría indicando que el ajuste obtenido es de buena calidad. El cuadrado de las diferencias entre el valor observado y el valor graduado impide que diferencias de distinto signo se anulen, produciendo sumas totales cercanas a cero (0), sin que necesariamente el ajuste sea de buena calidad.

El segundo término de la ecuación representa la calidad del suavizamiento, y corresponde a las diferencias finitas de orden " $z$ ", comúnmente tres (3) o cuatro (4), de los valores graduados. Se concluye que el suavizamiento en los valores graduados ha sido alcanzado cuando la suma de sus diferencias finitas tiene similar objetivo que en el caso anterior. Resulta importante mencionar que esta sumatoria de diferencias finitas no tiene significado en sí misma, pero resulta de utilidad al querer comparar los grados relativos de suavizamiento en diferentes graduaciones de los mismos datos y también con los datos sin graduar.

En el segundo término el parámetro $h$ es un número real positivo, que permite controlar el énfasis dado a $F$ y $S$ en la minimización de $M$. Comúnmente la minimización se conoce como la graduación de Whittaker tipo B. El Tipo A corresponde al caso particular del Tipo B con $w_{x}=1$, para todo $x$. A pesar que pueden utilizarse diferentes métodos para minimizar los dos tipos de graduación, se usará un método común para ambos, teniendo en consideración que el Tipo A está incluido dentro del Tipo B.

En la determinación de los pesos $w_{x}$ se debe considerar que se desea minimizar el valor de $M$ utilizando los valores observados, por lo cual resulta natural dar más importancia a aquellas edades donde existan más observaciones y donde las dispersiones sean menores, según esto una opción de valor para los pesos se determina mediante:

$$
w_{x}=\frac{n_{x}}{v_{x}\left(1-v_{x}\right)}
$$

Sin embargo, para determinar los pesos $w_{x}$ es preferible que no dependan de los valores graduados $v_{x}$, pues en ese caso, la minimización de $M$ estaría dificultada por una selección algebraicamente inadecuada de los pesos, por lo cual resulta preferible utilizar $u_{x}$ en lugar de $v_{x}$ de modo que los pesos quedan:

$$
w_{x}=\frac{n_{x}}{u_{x}\left(1-u_{x}\right)}
$$

Cuando los pesos en $M=F+h S=\sum_{x=1}^{n} w_{x}\left(v_{x}-u_{x}\right)^{2}+h \sum_{x=1}^{n-z}\left(\Delta^{z} v^{x}\right)^{2}$ se toman iguales al tamaño de la muestra $n_{x}$, los valores de $F$ resultan muy grandes, por lo cual se debe 
determinar un valor de $h$ adecuado para equiparar el énfasis dado al ajuste en menoscabo del suavizamiento. Una forma de disminuir dicho efecto es dividir el tamaño de la muestra por el promedio aritmético de los tamaños de la muestra en todas las edades $x, \bar{n}=\frac{\sum_{x=x_{1}}^{x_{2}} n_{x}}{\left(x_{2}-x_{1}\right)}$ quedando los pesos como:

$$
w_{x}=\frac{n_{x}}{\bar{n}}
$$

Con esta definición de los pesos se mantiene la proporcionalidad deseada para determinar el ajuste, dando más importancia a aquellas edades que tienen más observaciones sobre las que tienen menos datos. Además permite elegir un valor pequeño de $h$ para la determinación del suavizamiento. Una vez definido lo anterior, al utilizar el metodo $\mathrm{W}-\mathrm{H}$ en las estimaciones de edades de 0-80 años, obtenemos la siguiente gráfica:

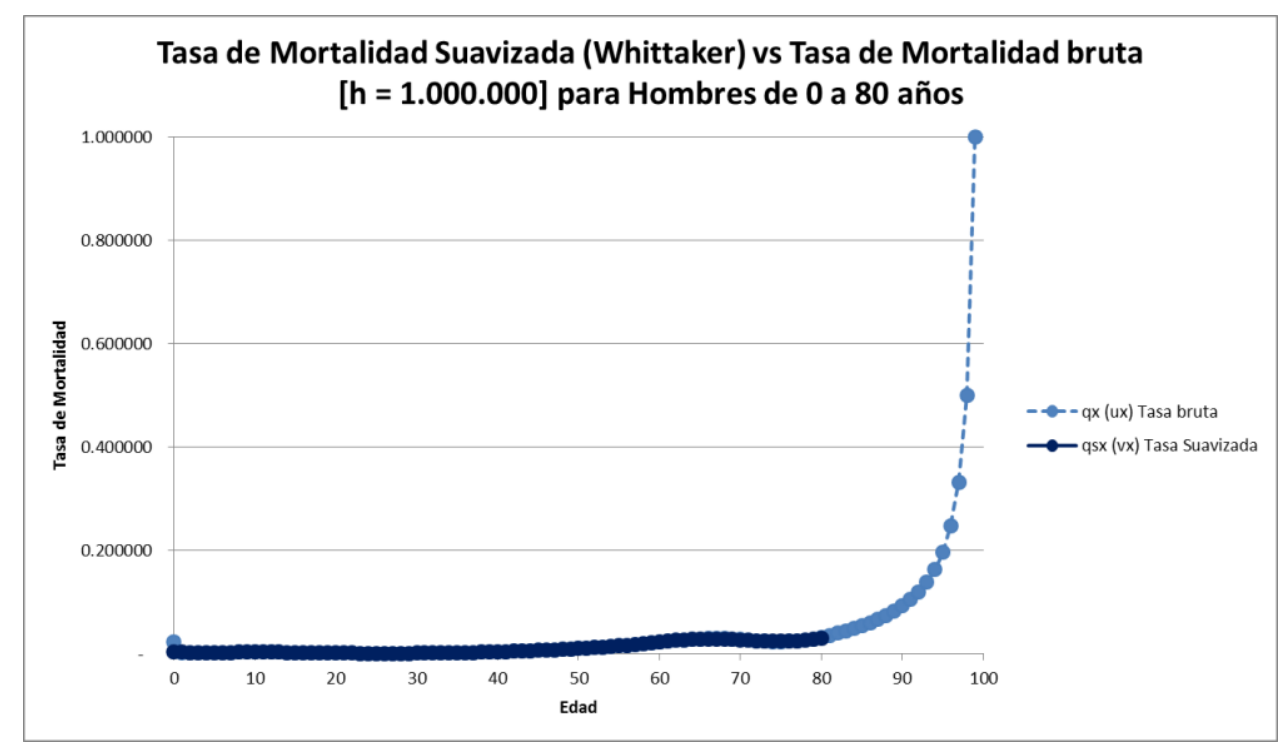

Figura 4: Comparación de las tasas de mortalidad estimada y suavizada por W-H.

Como era de esperarse, el método $\mathrm{W}-\mathrm{H}$ prácticamente describe la misma curva que las estimaciones por el spline, porque las variaciones entre cada intervalo producida por la interpolación son muy suaves, por lo tanto, la corrección del método es mínima. Como se planteó anteriormente los métodos de graduación buscan reducir las diferencias entre los intervalos para tener curvas suaves, pero al realizar la interpolación de splines, tiene la ventaja que las curvas que se obtienen tienen muy pocas variaciones y requieren correcciones muy pequeñas. 


\section{Conclusiones y Reflexiones Finales}

La tabla de mortalidad permite describir el comportamiento de la mortalidad por edades, lo cual es de importancia desde que la mortalidad es diferencial según la edad. Permite obtener probabilidades y otras medidas convencionales de la mortalidad, que son más apropiadas que las tasas de mortalidad por edad, sea para calcular los sobrevivientes de una población, para combinarlas con probabilidades de otros grupos de edades, o para derivar relaciones analíticas entre las diversas variables demográficas. El modelo de tablas de mortalidad puede ser usada en el análisis de diversas características socioeconómicas de la población, tales como la fuerza de trabajo, la población en edad escolar y la regulación de los sistemas de jubilación y pensiones para las personas de la tercera edad.

En la investigación se presenta un ejemplo de tablas de mortalidad para hombres, tratando de representar lo mejor posible, con base en la información disponible, la mortalidad asociada a las diferentes edades de la población observada, en este caso Honduras. La tabla presentada esta elaborada con los supuestos de tablas utilizadas para seguros de vida, ya que las tablas para rentistas consideran principalmente a las poblaciones que cotizan en algún plan de jubilación, para poder determinar las probabilidades de muerte de ese grupo y las probabilidades de incapacitarse a diferentes edades. El método puede ser utilizado en este tipo de tablas, pero los indicadores básicos para las estimaciones deben ajustarse para representar las probabilidades asociadas a este grupo.

Las tablas de mortalidad son una variable esencial para la determinación de las reservas para las pensiones como de los recursos requeridos para mantener el principio básico de la sostenibilidad financiera del sistema en el mediano y largo plazo. Por eso la importancia que las tablas representen lo más certeramente posible las probabilidades asociadas a la población donde se utilizan, y no solamente la utilización de tablas internacionales. También la actualización periódica de tablas es necesaria, por ejemplo, Colombia ya tiene nuevas tablas para rentistas y desde 2010 dejo de utilizar las tablas de rentistas 80-89, y estados unidos hace una actualización constante de sus tablas de mortalidad, teniendo tablas para diferentes grupos y con diversas características.

El método de estimación de tablas presentado en esta investigación, no pretende sustituir los métodos convencionales de elaboración de tablas de mortalidad, es simplemente para estimar las tablas cuando se dispone de poca información. Pero se espera que en el mediano plazo el país tome conciencia de la importancia de las mismas, y trabaje con todas las organizaciones para la recolección de información adecuada y poder elaborar las tablas de la forma convencional que resulta ser la que mejor describe las probabilidades del grupo en observación. Si los gobiernos desean realmente estimular una mejora social para las personas 
en edad de retiro, y que las personas mayores tengan jubilaciones adecuadas es importante que se elaboren tablas adecuadas para garantizar la estabilidad del sistema.

\section{Bibliografía}

American Psychological Association. (2013). Apastyle. Obtenido de http://www.apastyle.org/

Anderson, Black, Hair, \& Tatham. (1999). Análisis Multivariante. Prentice Hall.

Bannister, J. (1997). Insurance solvency analysis. LLP limited, second edition.

Boj del Val, E., Claramunt Bielsa, M., \& Fortiana Gregori, J. (2004). Análisis multivariante aplicado a la selección de factores de riesgo en la tarificación. Cuadernos de la Fundación MAPFRE Estudios, № 88.

Cardozo, F., \& Zarruk, A. (2010). Nuevas tablas de mortalidad de rentistas. Fasecolda, 2024.

CEPAL - Naciones Unidas. (18 de July de 2015). CEPAL Datos y estadísticas. Obtenido de Comisión Económica para América Latina y el Caribe: http://www.cepal.org/es/datos-y-estadisticas

Comisión Nacional de Bancos y Seguros . (1 de September de 2014). CNBS. Obtenido de Comisión Nacional de Bancos y Seguros : http://www.cnbs.gob.hn/files/circulares/2004/C0032004.htm

Comisión Nacional de Bancos y Seguros. (1 de September de 2015). CNBS CIRCULAR CNBS No.003/2004. Obtenido de RESOLUCIÓN No.041/13-01-2004. Comisión Nacional de Bancos y Seguros: http://www.cnbs.gob.hn/files/circulares/2004/C0032004.htm

De Vicente, M., Manera Bssa, J., \& Blanco, F. (2000). Análisis Multivariante para las Ciencias Sociales. Madrid: Dykinson.

FundaciónMAPFRE. (28 de August de 2014). MAPFRE. Obtenido de DICCIONARIO MAPFRE DE SEGUROS: http://www.mapfre.es/wdiccionario/general/diccionariomapfre-seguros.shtml

Garrido, \& Comas. (1987). Teoría general y derecho español en los seguros privados. En Tratado general de seguros: teoría y práctica de los seguros privados. Editado por el Consejo General de los Colegios de Agentes y Corredores de Seguros de España. 
Hossack , I., Pollard , J., \& Zehnwirth, B. (1999). Introducción a la estadística con aplicaciones a los seguros generales. Editado por Fundación MAPFRE Estudios en 2001.

IHSS. (2004). Boletín Estadístico.

INE. (12 de April de 2015). Instituto Nacional de Estadistica. Obtenido de INE Web site: http://www.ine.gob.hn/drupal/

Lowrie, W. B. (1993). Multidimensional Whitaker-Henderson Graduation With Constraints and Mixed Differences. Transaction of Society Actuaries, 45, 215-255.

Macaulay, F. R. (1931). The Smoothing of Time Series (Vols. 0-87014-018-3). D.C.: NBER.

Mazzeo, V. (2014). Demografia Social I. Buenos Aires: Universidad de Buenos Aires.

Naciones Unidas. (16 de April de 2014). Bienvenido a las Naciones Unidas. Obtenido de Declaracion Universal de los derechos Humanos : http://www.un.org/es/documents/udhr/

Nieto, U., \& Vegas, J. (1993). Matemática actuarial. Editorial MAPFRE S.A.

Secretaría de Salud [Honduras], Instituto Nacional de Estadística e ICF International. (2013). Encuesta Nacional de Salud y Demografía 2011-2012. Tegucigalpa: SS, INE e ICF International.

Secretaria de Salud. (2013). "Por una Honduras saludable" Modelo Nacional de Salud. Tegucigalpa: Secretaria de Salud.

Shapiro, A. (2001). Soft Computing Applications in Actuarial Science. Obtenido de http://www.soa.org:80/library/arch/2000-09/arch01v113.pdf

Superintendencia de Banca, Seguros y AFP - Republica del Peru. (15 de September de 2015). Superintencia de Banca y Seguros. Obtenido de Proyectos en Implementación: http://www.sbs.gob.pe/principal/categoria/implementacion-de-taxonomia$\mathrm{xbrl} / 1124 / \mathrm{c}-1124$

SVS. (20 de September de 2015). Superintendencia de Valores y Seguros. Obtenido de http://www.svs.cl/portal/principal/605/w3-channel.html

World Health Organization . (2000). World Health Report. 
World Health Organization. (25 de September de 2015). Global Health Observatory Data Repository. Obtenido de Adult mortality Data by country: http://apps.who.int/gho/data/node.main.111?lang=en

"LA REVISTA INNOVARE NO SE HACE RESPONSABLE EN NINGÚN CASO DE LOS CONTENIDOS, DATOS, CONCLUSIONES U OPINIONES VERTIDAS EN LOS ARTÍCULOS PUBLICADOS, SIENDO ESTA RESPOSABILIDAD EXCLUSIVA DEL (DE LOS) AUTOR (AUTORES)" 